\title{
Piezoelectric Rainfall Energy Harvester Performance by Advanced Arduino based Measuring System
}

\author{
G. Acciari, M. Caruso, R. Miceli, L. Riggi, P. Romano, G. Schettino, F. Viola
}

Dipartimento di Energia, ingegneria dell'Informazione e modelli Matematici, DEIM

University of Palermo, Palermo, Italy

\begin{abstract}
This paper presents the performances of rainfall energy harvesting through the use of a piezoelectric transducer and an Arduino-based measuring system. Different studies agree on the possibility of generating electricity from rainfall, but to date, a study on measuring the quantity of energy produced during rainfall is still missing. The present study begins with results obtained from laboratory researchers using piezoelectric transducers and oscilloscopes, finalized to measure the energy produced from a single raindrop, and concludes with an ad hoc Arduino-based measuring system, aimed to measure the actual amount of electrical energy produced by a piezoelectric transducer that is exposed to rainfall of variable durations.
\end{abstract}

\section{Index Terms - Energy harvesting, piezoelectric sensor}

\section{INTRODUCTION}

Research in the field of electrical engineering has been long engaged in the identification of new forms of energy reduction from common and everyday life sources. One of the latest techniques in this exploration phase is the conversion of mechanical energy, otherwise dispersed, into electricity by the employment of harvesters. This conversion creates micro-productions of electrical energy caused by vibrations of a piezoelectric transducer, which is then used to feed sensors, or electronic instruments, with small energy consumption (e.g., wireless sensors used for monitoring applications, which still need chemical batteries and have high costs of maintenance and disposal).

Piezoelectric materials have a lack of symmetry in the crystal lattice, leading to a dipole moment generation, which is also function of the stress acting on the structure. The lattice structure allows the fabrication of devices both in the macro and in the micro scales, overcoming the limitations due to the number of windings to be used in the electromagnetic transducers.

It can be stated that the kinetic energy of the ambient vibrations, which represents an energy normally wasted in the environment without a useful purpose, can be coupled to electromechanical transducers in order to feed electronic monitor apparatus. The vibration energy can be regarded as marginal energy due to its small entity, but it can be suitably adopted for utilities of reduced energy capacity, such as the transmission of information.

A complete overview on the possibility of harvest energy in different scenarios is difficult to be provided, since novel approaches and innovative ideas are continuously being developed: Benasciutti [1] studied the possibility of harvesting energy from vibrating shoe-mounted piezoelectric cantilevers, whereas Xiang et al. [2] faced the harvesting of energy induced from the deformation of sidewalks due to moving vehicles. Moreover, Van den Ende et al. [3] discussed about the harvesting from automotive tires and Hobeck [4] proposed an innovative piezoelectric grass energy harvester. In addition, Jager et al. faced the rainfall energy harvest as well [5-6], with the idea of converting the kinetic energy possessed by the drops of rainwater into electrical energy by means of piezoelectric plates. A pioneering comparison of different piezoelectric materials finalized to investigate the possibility of energy generation water droplets energy sources for low power electronic devices was studied by Vatansever et al. [7].

These studies confirmed that the single drop of water hitting the piezoelectric plates generates voltages less than a dozen of volts (peak to peak), but no evaluation on power has been proposed. The drops of rain strike the piezoelectric material in a cantilever configuration, which may be subject to study to improve the energy produced, as described by Benasciutti et al. [8-9]. Although this peak to peak voltage seems adequately high to interact with electronic devices or rectifiers, a more accurate characterization is required, in order to dispel excessive optimistic predictions. The concept of energy flow, presented by Liang in [10] and by Erturk [11], clarifies the dissipation of energy during the harvesting process, in order to separate the electromechanical coupling coefficient of the system, natural frequencies, damping ratio and electric load. Roundy [12] proposed useful schemes for the modeling of the electromechanical coupling and these models are here validated for the raindrop harvester. The French studies [5-6] have demonstrated that a single raindrop with diameters ranging between 1 and $5 \mathrm{~mm}$ can generate up to $12 \mathrm{~mJ} / \mathrm{s}$, whereas Italian studies have shown that the piezoelectric transducer-based conversion system produces $0.43 \mu \mathrm{J}$ of energy for each raindrop that impacts it [13-18]. A recent work of Helseth [19] introduces different possibilities to harvest raindrop energy with piezoelectric and triboelectric transducers and evaluates the energy potential due to rain parameters. Lin et al. [19] with a pioneering work, suggest a recover energy of $30 \mathrm{~nJ}$ of electrical energy due to a droplet of $30 \mathrm{~mL}$. These results leave some uncertainty on the actual capability to generate a sufficient amount of electrical energy from rain. 
The aim of this paper is to study the amount of power harvested from the rain, since such characterization is still missing in literature. In order to better define the amount of power extracted from a single drop of rain, different systems (consisting of commercial transducers) are compared. These systems are exposed to both natural and artificial rain and they are also subject to suitable configurations with different shape and deflection in order to increase the extracted power. Then, an experimental identification on the amount of energy obtainable from the impact of multiple drops on a piezoelectric transducer during a real rainfall is performed. For this purpose, an ad hoc measuring tool has been set-up, by exploiting the potential of the open source prototyping system named "Arduino". This study can lead to the evaluation of the energy of raindrop in order to create smart sensor that feeds itself and communicate the kinetic energy of raindrop to prevent soil erosion.

In order to give a complete overview of the raindrop harvesting two review can be taken into account $[21,22]$.

This paper is structured as follows: Section II describes the Arduino system, Section III describes piezoelectric harvesters. In Section IV a model of the cantilever is developed, whereas in Section $\mathrm{V}$ the developed model is used to evaluate the Arduino measuring system. Finally, the proposed system is used in an open field test, described in Section VI.

\section{HARDWARE PLATFORM AND CIRCUITAL COMPONENTS}

Arduino [23-24] is a hardware platform used for the development of applications based on ATMEL microcontrollers. The model "Arduino UNO R3 SMD", which is used in the current study, is a standard commercial board entrusted to acquiring and recording inputs. In this study, the Arduino UNO board was connected to the Ethernet Shield, in order to allow data storage on a microSD card (Samsung EVO HC 32GB, class 10), which was inserted into the Ethernet Shield and used to record data. The detection of values only between $0 \mathrm{~V}$ and $5 \mathrm{~V}$ was the only limit of the Arduino platform regarding the proposed study. In order to solve this problem, an ad hoc electrical circuit was used to regulate the signal, so that once the oscillation was exhausted, it guaranteed the voltage trend at $2.50 \mathrm{~V}$, as to provide, after an appropriate encoding of the obtained results, the entire transition from $-2.5 \mathrm{~V}$ to $+2.5 \mathrm{~V}$. The measurement set-up employing Arduino is shown in Fig. 1.

The circuit connected to the Arduino system is created by a low voltage rail-to-rail operational amplifier fed by the same board. This circuit works in the desired voltage range accepted by the Arduino board in order to optimize its work. The circuit is also comprised of four resistors used to set bias and gain.

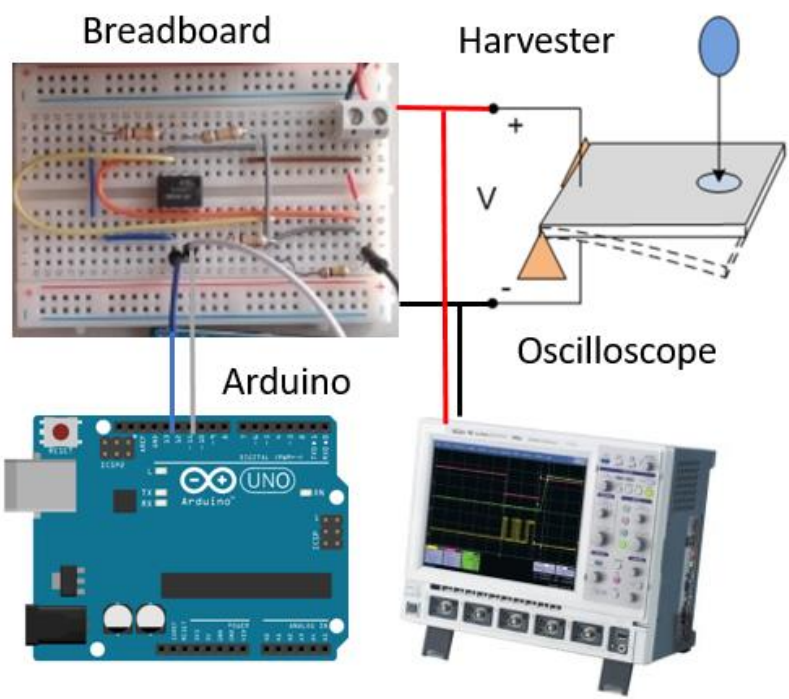

Fig. 1 -Measurement set-up employing Arduino.

\section{PIEZOELECTRIC TRANSDUCERS AND RAINDROP}

The ability of piezoelectric materials to convert electrical energy into mechanical and vice versa depends on their crystalline structure. The required condition for the piezoelectric effect is the absence of a center of symmetry in the crystal, which is responsible for charge separation between positive and negative ions and the formation of the Weiss domains. By applying an electric field to a piezoelectric material, the Weiss domains are proportionally aligned to the field. As a consequence, the size of the material changes, by increasing or decreasing if the direction of the Weiss domains is the same as or opposite to the electric field. Therefore, a stress (tensile or compressive) applied to a piezo-crystal will alter the separation between the positive and negative charge sites in each elementary cell, leading to a net polarization at the crystal surface. The piezoelectric effect verifies the first law of the thermodynamics (principle of conservation of energy).

In this study, the energy harvester consists of a piezoelectric film on an epoxy cantilever sandwiched between electrodes that are used to collect the generated power. A water drop falling on the structure creates an impulsive force that brings the internal lattice structure of the piezoelectric element to deform, causing the loss of symmetry, and therefore the generation of small dipoles, which global effect is an impulsive voltage on electrodes.

Mechanical vibrations follow the impact, stress is induced within the material, thus giving rise to an electrical source. A sheet of piezoelectric material has some limitations in the mechanical-electrical transduction for lowfrequency signals, since the effects of the induced electric field, generated in the hit region, are mitigated by the surrounding areas, and for large sheets effects are tenuous. The behavior of generated pulses depends on the state of locking of the piezoelectric film. 

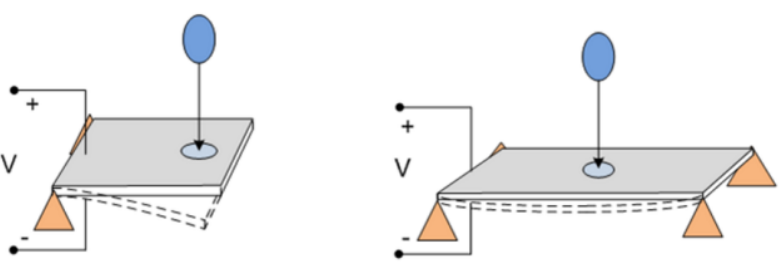

Fig.2. Piezoelectric cantilevers: one edge bound (left) and two edges (right).

Different studies in the literature show encouraging results with regards to the generation of electricity from water droplets [21,22]. The piezoelectric transducers can reach tens of volts, but this result does not yet allow to attribute to them the character of power generators. The water drops continuity in the same place is very variable: there may be intervals of seconds (small rainfall) or fractions of seconds (downpour). Different performances due to variable drops dimensions (mass) and impact point make it hard to model the phenomenon. The voltage has a peak waveform and not a continuous voltage, so an equivalent average voltage has to be defined. For a power system the equivalent average current can be obtained by using a bridge rectifier and a smoothing capacity (initially, for the theoretical model, this approach has been not considered).

Different transducers configuration can be adopted. For instance, Fig. 2 shows the two models locked in one end (Viola et al.,[13-18]) and two ends (Jager et al., [5-6]). Larger collection surfaces can be used, made with piezoelectric material (Perera et al., [26]), or with an inert collecting diaphragm linked to piezoelectric cantilevers (Wong Ching-Hong et al. [27]), as shown in Fig. 3.

In order to evaluate the output power of a piezoelectric transducer, the definition of a range of possible stresses is needed. The single drop of water can have a diameter that varies between 0.2 to $6 \mathrm{~mm}$. By considering a speed on impact of approximately $2 \mathrm{~m} / \mathrm{s}$ for the small drop and $9 \mathrm{~m} / \mathrm{s}$ for the largest one, it is possible to estimate the energy input as:

$$
E_{\min }=3.1 \mu \mathrm{J}, E_{\max }=0.063 \mathrm{~J}
$$

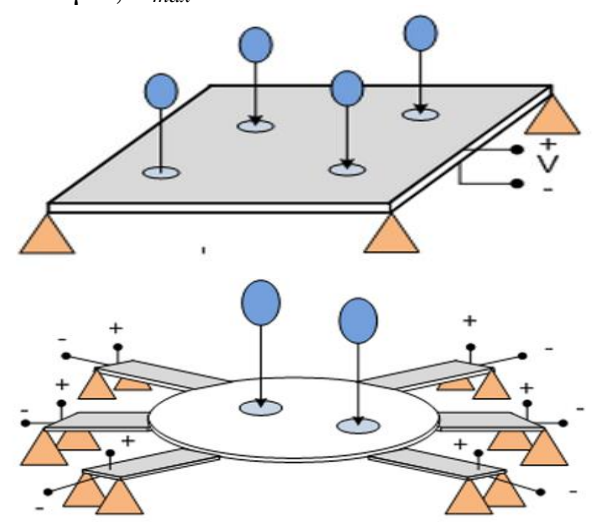

Fig. 3. Larger piezoelectric harvesting systems.
Furthermore, by considering an interval of 2 seconds between two consecutive drops, the power is given by:

$$
P_{\min }=1.5 \mu \mathrm{W}, P_{\max }=0.031 \mathrm{~W} \text {. }
$$

The harvestable power, however, is affected by several factors. The drop, while fully centering the piezoelectric film, is not able to transfer maximum energy as it is subject to the phenomenon of splashing: the collision is not complete since the main drop is separated itself into small drops, leaving the impact surface. Therefore, an efficiency of collision $\eta_{\text {collision }}$ must be introduced. In addition, the drop stresses the piezoelectric according to the 31 mode and not all the energy is converted into charges on the plates of the transducer. Therefore, the efficiency of the piezoelectric element $\eta_{\text {piezo }}$ should be added, as well as for $\eta_{\text {rect }}$ in order to take into account the losses of the rectifying bridge.

Thus, the output power is given by [22]:

$$
\mathrm{P}_{\text {out }}=\eta_{\text {collision }} \cdot \eta_{\text {piezo }} \cdot \eta_{\text {rect }} \cdot \mathrm{P}_{\max } \cdot
$$

The output power is certainly reduced. Therefore, the main purpose is to maximize it.

The transducers on which the experiments were conducted are the Mide Volture V22B [28] and the MEAS LDT1-028K [29] types, both mounted on a suitable support, as shown in Figs.4 and 5, respectively. The PVDF Meas is customable, the PZT Volture has a rigid structure and no modification can be introduced.

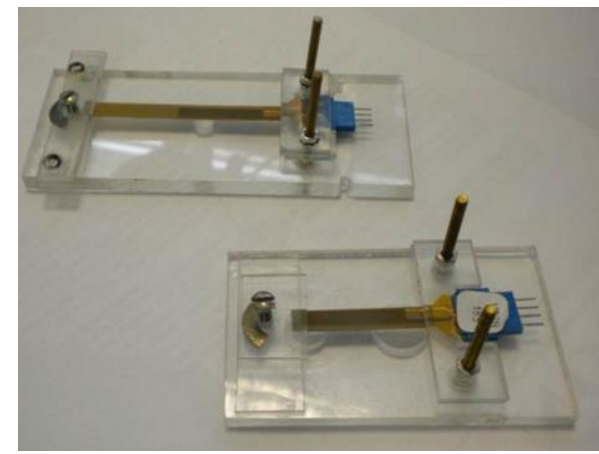

Fig. 4. Lead zirconate titanate (PZT) transducers: V22BL in the top and $V 22 B$ in the bottom of the picture.

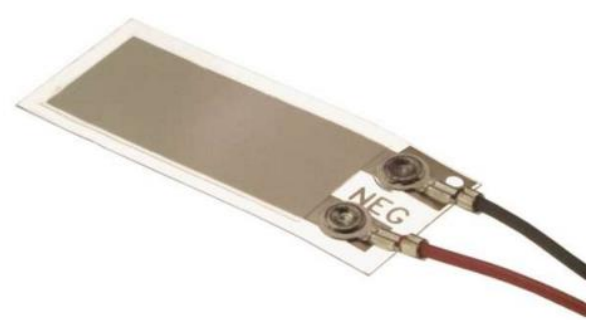

Fig. 5. Meas LDT1-028k polyvinylidene difluoride (PVDF) transducer. 


\section{CANTILEVER MODEL}

In order to evaluate the harvested power, which is taken by the employment of an AC-DC converter (composed by a rectifier bridge and a smoothing capacitor), it is necessary to study the shape of the waveform that interacts with the bridge.

Fig.6 reports the electro-mechanical scheme presented by Roundy and Wright [12]. The electrical and mechanical parts are connected via an unusual transformer, as demonstrated by Pinna et al. [30].

For a mode 31 , it is possible to define the following equations:

$$
\begin{aligned}
& S_{1}=s_{11} \sigma+d_{31} E_{3} ; \\
& D_{3}=d_{31} \sigma+\varepsilon E_{3},
\end{aligned}
$$

where the tensors depend on the direction of both the force and the field, $S$ is the strain, $s$ is the compliance coefficient, $\sigma$ is the stress, $d$ is the piezoelectric coefficient of strain, $E$ is the electric field, $D$ represents the displacement field and $\varepsilon$ the permittivity. Impact and following vibration are related to axis 3 .

In the mechanical scheme the stress $\sigma$ has the role of voltage; first derivative of strain, $\dot{S}$, that one of current. On the equivalent inductance $\mathrm{L}_{\mathrm{m}}$ the applied voltage is related to the second derivative of strain, on the equivalent resistance $R_{m}$ voltage is related to the first derivative and on the capacitance $C_{m}$ directly to the strain. The derivative is related to the longitudinal axis of the harvester, referred as axis1, not to time

In the mechanical part, the inductor $L_{m}$ represents the equivalent mass and the inertia of the vibrating mass, while $R_{m}$ represents the mechanical losses. In addition, $C_{m}$ represents the mechanical stiffness and the stress generator $\sigma_{\text {in }}$ is due to external mechanical vibration. In the electrical part, $C_{e}$ represents the capacitance of the piezoelectric element, $R_{e}$ an external load and $V_{e}$ is the voltage across the piezoelectric transducer. Electric and mechanical parameters depend on the shape and the vibration mode of the piezoelectric transducer.

By applying Kirchhoff's law on the first loop, the following equation is obtained:

$$
\sigma_{\text {in }}-L_{m} \ddot{S}-R_{m} \dot{S}-S / C_{m}=V_{m},
$$

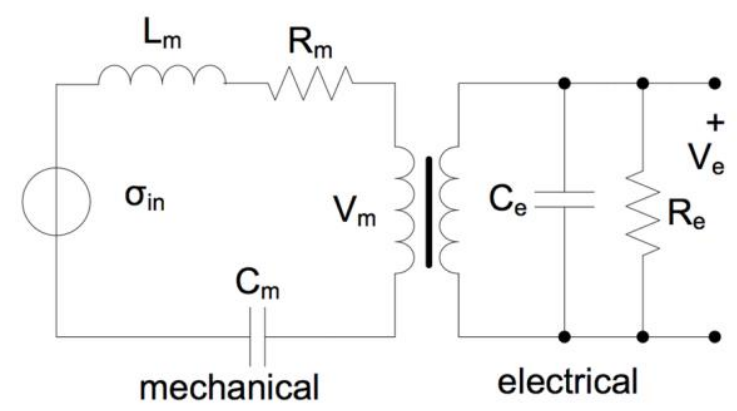

Fig. 6. Equivalent electro-mechanical scheme.

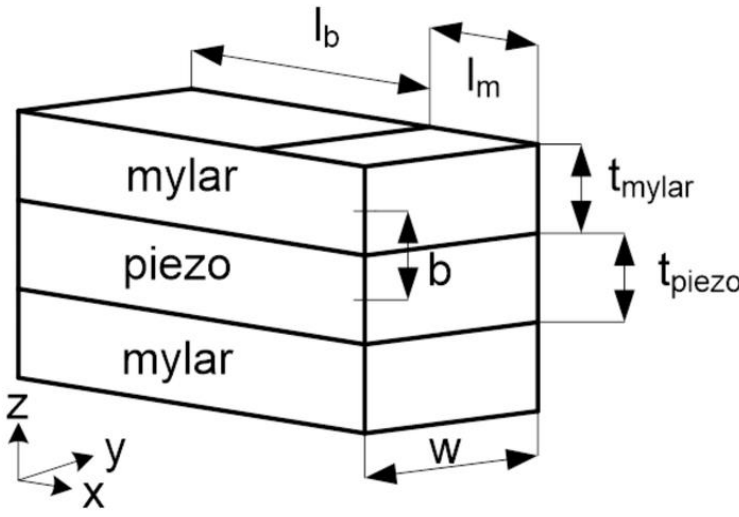

Fig. 7. Layer of the PVDF cantilever.

the equivalent transformer constitutive equation are:

$$
\begin{aligned}
& V_{m}=n_{v} V_{e}, \\
& I_{m}=\frac{1}{n_{i}} I_{e} .
\end{aligned}
$$

The cantilever configuration is studied for the PVDF transducer, which has a free edge. This harvester is composed by only one piezoelectric layer. The inertia moment of the mass is evaluated by the following equation:

$J=2\left[\frac{w t_{\text {mylar }}^{3}}{12}+w t_{\text {mylar }} b^{2}\right]+\frac{w t_{\text {piezo }}^{3}}{12}$,

where $\mathrm{b}$ is the distance between the middle points of layers, as shown in Fig 7.

The average stress in the beam is equal to:

$$
\sigma_{\text {in }}=\frac{1}{l_{b}} \int_{0}^{l_{b}} \frac{M(x) b}{J} d x,
$$

where:

$$
M(x)=F_{i n}\left(l_{b}+l_{m} / 2-x\right),
$$

$l_{b}$ is the length of the cantilever and $F$ is the force exerted by the drop.

$K_{l}$ is the geometrical coefficient that correlates the stress $\sigma_{i n}$ and $F_{\text {in }}$ :

$$
\sigma_{\text {in }}=K_{1} F_{\text {in }} \text {. }
$$

During the vibrations, $K_{l}$ relates also the stress due to the mass of water attached to the cantilever:

$$
\sigma_{L}=K_{1} F_{m}
$$


and in similar way it is related to the second derivative of the strain by:

$\sigma_{L}=K_{1} K_{2} m \ddot{S}$

where $m$ is the mass of the water and $K_{2}$ is a geometrical coefficient.

The equivalent inductance $L_{m}$ and the equivalent resistance $R_{m}$ are given by, respectively:

$L_{m}=K_{l} K_{2} m, R_{m}=K_{l} K_{2} b_{m}$

where $b_{m}=\sqrt{\frac{K}{m}} / \frac{m}{Q}$ is the damping factor, $K=1 / s_{11}$ is the Young's module and $\mathrm{Q}=10$.

The equivalent capacitance relates strain with stress by using the compliance constant $\mathrm{s}_{11}$ :

$\sigma_{C}=\frac{S}{s_{11}}=\frac{1}{C_{m}} \int \dot{S} d x$

In order to evaluate the voltage ratio $n_{v}$, a strein zero configuration is considered:

$$
0=s_{11} \sigma_{T}+d_{31} E_{3}
$$

The relationship between the electric field and the voltage can be expressed by the following equation:

$\sigma_{T}=-\frac{d_{31}}{s_{11}} \int E_{3} d z=-\frac{d_{31}}{s_{11}} \frac{V_{e}}{t_{\text {piezo }}}$,

$n_{v}=-\frac{d_{31}}{s_{11} t_{\text {piezo }}}$.

The electric current is equal to:

$i=\frac{V_{e}}{R}+C_{e} \dot{V}$,

where

$$
C_{e}=\varepsilon \frac{w l_{b}}{t_{\text {piezo }}}
$$

If the external electric field is neglected, then:

$$
D=\frac{d_{31}}{s_{11}} S,
$$

the electrode charge is given by: $q=w l_{b} D=w l_{b} \frac{d_{31}}{s_{11}} S$,

The current I and the current ratio are equal to:

$$
i=w l_{b} \frac{d_{31}}{s_{11}} \dot{S}=\frac{1}{n_{i}} \dot{S} .
$$

The characterization has been carried out for a one-edge fixed cantilever, but the two-edge cantilever can be also studied in a similar manner.

The transfer function can be easily developed, where the mechanical and electrical equations are given by:

$$
\begin{aligned}
& L_{m} \ddot{S}+R_{m} \dot{S}+S / C_{m}=\sigma_{i n}+V_{m}, \\
& w l_{b} \frac{d_{31}}{S_{11}} \dot{S}=\frac{V_{e}}{R}+C_{e} \dot{V},
\end{aligned}
$$

by transforming in the Laplace domain:

$$
\begin{aligned}
& S\left(s^{2}+\frac{b_{m}}{m} s+\frac{1}{m K_{1} K_{2} K}\right)=\frac{\sigma_{\text {in }}}{K_{1} K_{2} m}+\frac{d_{31} K}{t_{\text {piezo }} m K_{1} K_{2}} \\
& S=\frac{\varepsilon}{t_{\text {piezo }} d_{31} K s}\left(s+\frac{1}{R_{e} C_{e}}\right) V .
\end{aligned}
$$

The voltage transfer function can be expressed as:

$$
V=\frac{A_{1} s}{s^{3}+B_{2} s^{2}+B_{1} s+B_{0}}
$$

where

$$
\begin{aligned}
& A_{1}=\frac{t_{\text {piezo }} d_{31} K \sigma_{\text {in }}}{\varepsilon m K_{1} K_{2}} \\
& B_{2}=\frac{b_{m}}{m}+\frac{1}{R_{e} C_{e}},
\end{aligned}
$$

$B_{1}=\frac{1}{m K_{1} K_{2} K}+\frac{b_{m}}{m}-\frac{d_{31}^{2} K^{2}}{\varepsilon m K_{1} K_{2}}$,

$B_{0}=\frac{1}{R_{e} C_{e} m K_{1} K_{2} K}$.

The magnitude of the Bode diagram is reported in Fig. 8. It can be observed that the maximum is located for $\omega_{n}=260$ $\mathrm{rad} / \mathrm{s}$. Such behavior is principally due to the mechanical properties of the cantilever. 


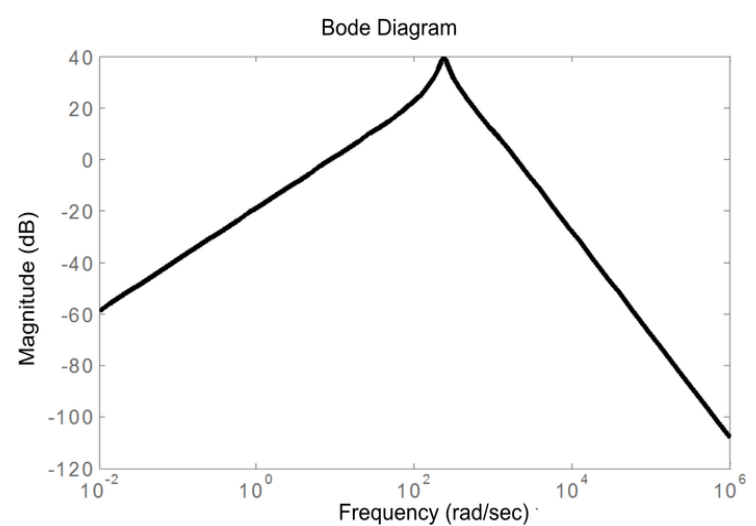

Fig. 8. Bode's diagram of the voltage transfer function.

\section{VALIDATION OF THE INSTRUMENT}

An indoor experiment under simplified conditions has been carried out in order to validate the proposed model and the transfer function. This test reconstructs the falling of water drops on the cantilever. Afterwards, the recorded output voltage is compared with the values obtained with the model. A pipette has been adopted for the rainfall simulation; this solution allowed the artificial creation of a calibrated water drop (with a default speed caused by the height of the fall).

The piezoelectric plate was fixed to a plane by means of a vise, so that the structure was stable on one side and free to move at the other. In this manner, the oscillatory properties of the material were kept unchanged, reproducing the behavior of a cantilever beam. The connectors of the lamina have been linked to a probe of the oscilloscope LT342L (Lecroy Inc.).

The pipette consists of a cylindrical capillary with thick walls, the end of which is cut along a section, as shown in Fig. 9, where the drops are formed.

The relationship between the droplet radius and the capillary radius is given by the following equation:

$R_{\text {drop }}=\left(\frac{3 R_{\text {capillary }} \gamma}{2 g \rho}\right)^{\frac{1}{3}}$

where $\rho$ is the surface tension of water, $\gamma$ is the density of water and $g$ is the gravity acceleration.

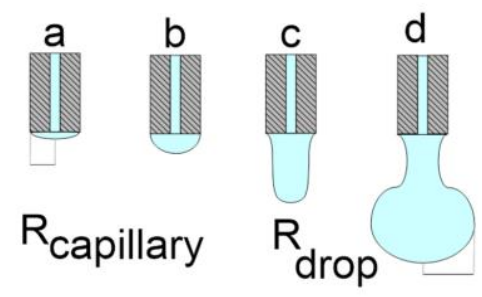

Fig. 9. Schematic of the release stages of the drop from the pipette.

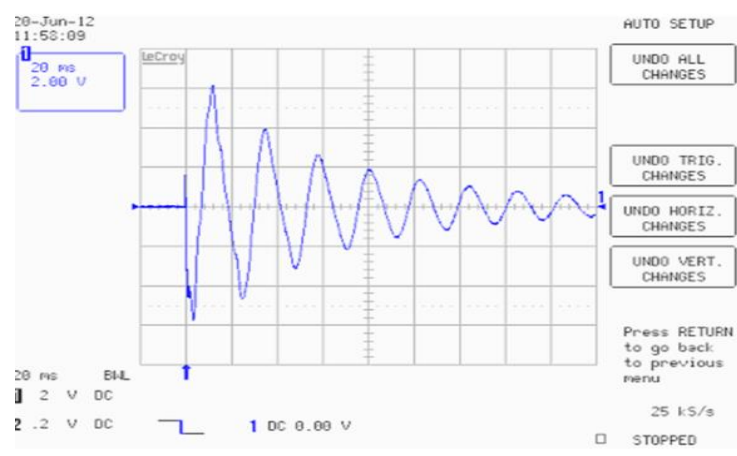

Fig. 10. Time profile of the recorded voltage.

However, the adoption of the capillary for the creation of the droplet has a drawback concerning the movement of the liquid through the capillary, due to the fact that the droplet ejection must be relatively slow, so that a certain condition of static equilibrium can be maintained. The employment of a disdrometer allows the achievement of better performances for the water droplets characterization.

A first test has been carried out considering a height of 80 $\mathrm{cm}$ and the waveform of the generated voltage detected by the scope (set to $2 \mathrm{~V} /$ division) is shown in Fig. 10.

As a pulse stress the system, the system itself responds to the frequency that maximizes the output. A maximum of $6 \mathrm{~V}$ has been reached, this is due to the precision of fall in the edge area of the cantilever, to the use of a very large drop, to the absence of the phenomenon of splashing.

The response of the model can be carried out by means of Simulink ${ }^{\circledR}$ program, in which a simple block diagram is realized and depicted in Fig. 11 (a).

The stress due to the water drop can be simulated by an impulsive force and, therefore, a similar function to the Dirac delta function is considered in this study. Fig. 11 (b) shows the time profile of the pulse, which is defined as the limit for $\Delta$ that tends to zero of the rectangular function with height equal to $1 / \Delta$ and amplitude $\Delta$. For $\mathrm{t}=\mathrm{t}_{0}$, this function can have an infinite value, while outside the interval $\left[\mathrm{t}_{0}\right.$, $\left.\mathrm{t}_{0}+\Delta\right]$ the function value is equal to zero.

By considering an observation time equal to $10 \mathrm{~s}$, the pulse generator has been considered as not ideal and the impact of the drop on the piezoelectric has been set equal to $0.08 \%$ of the time interval. The mass is $0.12 \mathrm{~g}$, while the final velocity and the force are set to $1.24 \mathrm{~m} / \mathrm{s}$ and $0.77 \mathrm{~N}$, respectively.

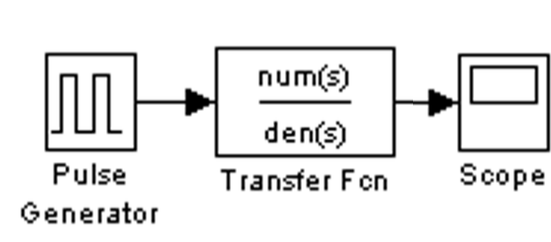

(a)

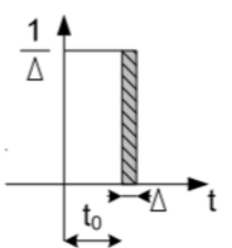

(b)
Fig. 11. Simulink model of the piezoelectric system (a). Time-profile of the stress caused by the water drop $(b)$ 


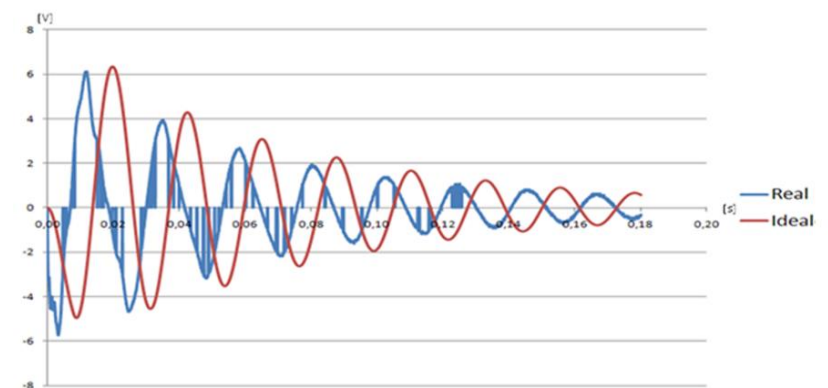

Fig. 12. Comparison between the time profiles of recorded and simulated voltage.

The comparison between the recorded voltage and the simulated one is shown in Fig. 12 and no splashing phenomenon has been observed.

Oscillations in the simulated system are very similar to the recorded ones, as well as for the voltage values.

Other laboratory tests with different height of fall of the drop have been carried out, demonstrating that, by increasing the height, the maximum value of the voltage produced by the drop is increased, keeping constant its geometrical dimensions. However, it has been also found that as the speed of fall increases, the splash phenomenon occurs, and a loss of mechanical energy causes a divergence of the theoretical results compared to experimental ones.

\section{EXPERIMENTAL STUDY}

Samplings were performed on January $18^{\text {th }}, 2015$, in San Cataldo (CL, Sicily, Italy), and on January $22^{\text {nd }}, 2015$, in Caltanissetta, (CL, Sicily, Italy). Arduino UNO, equipped with the Ethernet shield and breadboard with resistors and an integrated circuit, as shown in Fig. 13 (a), was located in a polystyrene waterproof box (see Fig. 13 (b)) to protect the circuit from the rain. The piezoelectric transducer was exposed to the meteorological phenomena through a gap suitably created in the waterproof box.

The measuring instrument was laid on the ground and it was free from any obstacles that could have limited the exposure to the rain. The results detected during the first sampling day at 05:45 pm.

The open field test showed different behaviors from those taken in the laboratory or by means of the mathematical model.

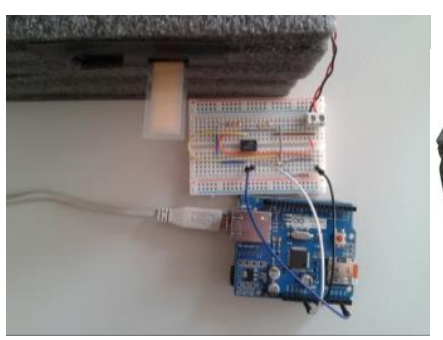

(a)

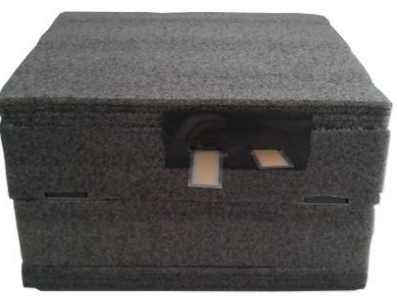

(b)
Fig. 13-Instrumentation adopted for the experimental study (a) and waterproof box used for the tests $(b)$.
The magnification of the oscillation caused by a single raindrop that reached a greater amplitude during the sampling is also shown in Fig 14 (b). The charging of energy is denoted by the unusual zig-zag trend at the beginning of the transient. This is precisely the moment when the drop collided with the piezoelectric wafer. Fig. 14 shows a little ripple for the recorded voltage, but not for the simulated one. In the inner laboratory test, no splashing was observed.

During the measurements, which lasted 90 seconds, the maximum voltage peak was $3.62 \mathrm{~V}$, the minimum was equal to $1.36 \mathrm{~V}$, with a range of $2.26 \mathrm{~V}$. The average value was equal to $2.463 \mathrm{~V}$, whereas the median and the mode values were equal to $2.48 \mathrm{~V}$ and $2.50 \mathrm{~V}$, respectively, with the standard deviation of 0.054 .

With careful examination of the graph shown in Fig. 15, small peaks can be noticed, which may have been caused by water drops that had lightly touched the piezoelectric transducer or by vibrations of the polystyrene box caused by the percussion of the rain or by splashing. Some of these peaks are shown with zoom in the same figure.
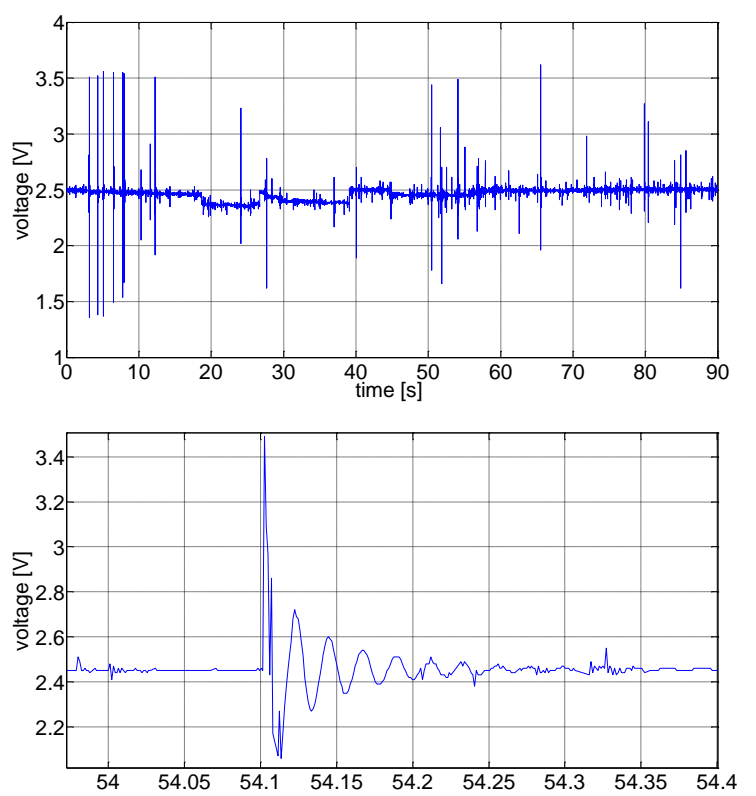

Fig. 14 - (a) Sampling of 90" performed outdoor in San Cataldo (CL, Italy), during the rainfall of January $18^{\text {th }}, 2015$, at 05:45 pm and (b) transient of $0,15 \mathrm{~s}$ caused by a single raindrop.

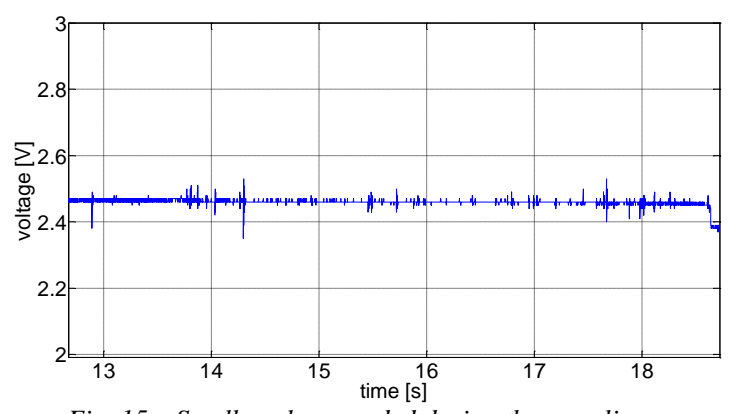

Fig. 15 - Small peaks recorded during the sampling 
The analysis of the data shows that the reference value during the lab tests were mainly $2.52 \mathrm{~V}$, and could rarely be noticed at $2.51 \mathrm{~V}$. Instead, the magnification of Fig. 14 demonstrates that the reference value continuously oscillates between $2.51 \mathrm{~V}$ and $2.49 \mathrm{~V}$. This could be caused by the wind or by rain that fell in the proximity of the sampling equipment, causing light vibrations. It is possible to establish a measurement error of $\pm 0.010 \mathrm{~V}$. During the sampling, the rainfall was of high intensity with thunder and wind and, from what can be deduced in the diagram, there were several drops that fully hit the center of the wafer. The power generated in this time frame is $P=2.916 \times 10^{-8} \mathrm{~W}$.

The first sampling instants are reported in Fig. 16. In this short time interval, when several raindrops landed, the graph denotes a decreasing trend (last quarter of the sampling). These drops always tilted towards the transducer, slipping across its surface and causing a continuous variation of the potential difference, not allowing the maintenance of the reference voltage value. Furthermore, in different diagram zones, a net variation of the reference voltage value (detail shown in Fig. 17) is observed. A plausible explanation could be the fact that some drops, once they hit the piezoelectric transducer, did not fall, thus causing a change in the mass of the transducer, so it was always subjected to pressure, despite there being drops that caused small and large oscillations during the considered time frames. The initial conditions were restored as soon as the collected drops fell.

In order to try to explain the found behavior, the mathematical model of section IV has been used. The parameters of the raindrop have been considered and Fig. 18 shows the response of a raindrop with mass of $0.12 \mathrm{~g}$ and final velocity of $1.24 \mathrm{~m} / \mathrm{s}$.

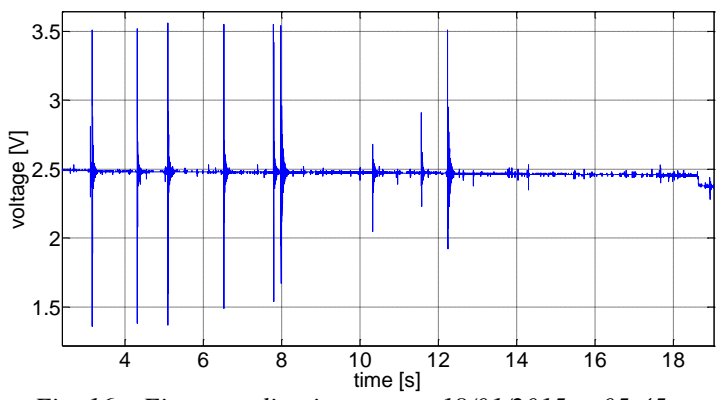

Fig. 16 - First sampling instants on 18/01/2015 at 05:45 pm.

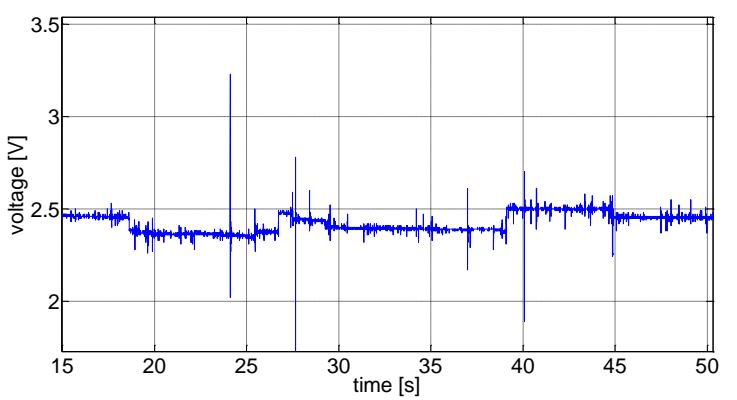

Fig. 17-Variation of the reference voltage on 18/01/2015 at 05:45 pm.

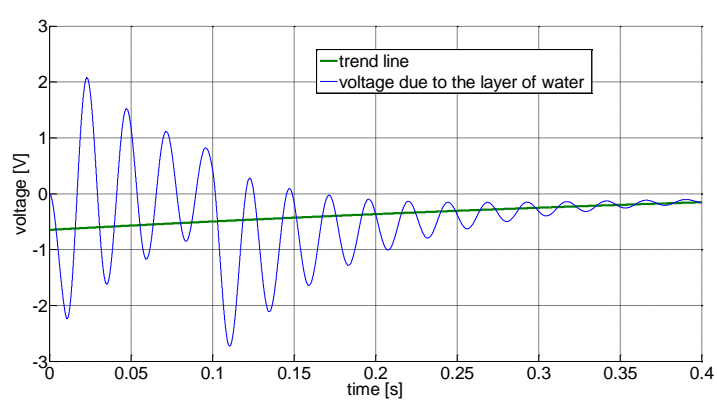

Fig. 18-Simulation of the cantilever with a layer of water, oscillations fluctuate around a trendline.

In equation (27) a constant stress has been added $\left(\sigma_{\text {avg }}\right.$, equal to a quarter of the raindrop stress) in order to reply the not fallen drop. Oscillations are not developed around the zero voltage, but are shifted toward negative values.

The data recorded during the tests of January $18^{\text {th }}$ and January $22^{\text {nd }}$ are all summarized in Table I.

Table. I-Main data recorded during the experimental tests.

\begin{tabular}{|c|c|c|c|c|c|c|c|}
\hline \multicolumn{8}{|c|}{ January $18^{\text {th }}$} \\
\hline hour & $\mathbf{t}$ & $\underset{n}{\text { Vmi }}$ & $\begin{array}{c}\text { Vma } \\
x\end{array}$ & $\begin{array}{c}\text { Vme } \\
\text { d }\end{array}$ & Me & $\Delta$ & Pmed \\
\hline $08: 50$ & $60 "$ & 1.51 & 3.61 & 2.504 & 2.50 & 0.0144 & $\begin{array}{c}2.086 \mathrm{x} \\
10^{-9}\end{array}$ \\
\hline 09:00 & $\begin{array}{c}120 \\
, "\end{array}$ & 1.99 & 2.91 & 2.50 & 2.50 & 0.00481 & $\begin{array}{c}2.39 \mathrm{x} \\
10^{-10}\end{array}$ \\
\hline $09: 30$ & $\begin{array}{c}300 \\
"\end{array}$ & 2.20 & 2.88 & 2.508 & 2.51 & 0.00474 & $\begin{array}{c}2.252 \mathrm{x} \\
10^{-10}\end{array}$ \\
\hline $09: 37$ & $\begin{array}{c}300 \\
"\end{array}$ & 1.72 & 3.59 & 2.487 & 2.50 & 0.02092 & $\begin{array}{c}4.379 \mathrm{x} \\
10^{-9}\end{array}$ \\
\hline $17: 45$ & $90 "$ & 1.36 & 3.62 & 2.463 & 2.48 & 0.054 & $\begin{array}{c}2.916 \mathrm{x} \\
10^{-8}\end{array}$ \\
\hline \multicolumn{8}{|c|}{ January $22^{\text {nd }}$} \\
\hline hour & $\mathbf{t}$ & $\begin{array}{c}\text { Vmi } \\
\text { n }\end{array}$ & $\begin{array}{c}\text { Vma } \\
x \\
\end{array}$ & $\begin{array}{c}\text { Vme } \\
\text { d }\end{array}$ & Me & $\Delta$ & Pmed \\
\hline $12: 50$ & $90 "$ & 1.72 & 3.42 & 2.509 & 2.51 & 0.01007 & $\begin{array}{c}1,015 \mathrm{x} \\
10^{-9}\end{array}$ \\
\hline $13: 30$ & $90 "$ & 2.33 & 2.91 & 2.51 & 2.51 & 0.00401 & $\begin{array}{c}1.607 \mathrm{x} \\
10^{-10}\end{array}$ \\
\hline
\end{tabular}

\section{INTERFACING CIRCUIT}

It appears evident that the same piezoelectric device may present different values of the extracted power: a harvester can produce power in the order of $\mathrm{mW}$ if attached to industrial motors, while the power decreases in the order of $\mu \mathrm{W}$ if the same device is attached to a human body. Similar considerations can be carried out for solar-powered sensors, which can benefit of power in the order of tens of $\mathrm{mW}$ for outdoor environments, while for indoor locations the power is in the order of $\mu \mathrm{W}$. Therefore, it is clear that the sensors applied in locations without any energy supply require a higher level of care in terms of their design.

Moreover as stated in [22], the harvesting from rain is considered an interesting of ecological source of renewable energy, and available kinetic energy of rainfall is not trifling in tropical countries at the equators.

A piezoelectric beam in any cantilever configuration, and a storage system makes the raindrop energy harvester, 
whose scheme is reported in Fig. 19. A rectifier bridge, which is needed because of the alternate trend of the voltage waveform and which employs low-loss full-wave bridge rectifier for the voltage consumption reduction, and several capacitors, composes the energy storage. A micro power step-up low voltage booster module is used with the purpose of obtaining both a better energy storage and a suitable voltage. A capacitor receives the pulses generated by the rain and it progressively charges until it reaches the voltage values suitable for both the sensors and the transmission system supply. The global performances of the harvesting system depend on the AC-to-DC converter, acting as an interface circuit.

The ultra-low power microcontroller regulates both the booster and the energy management. Fig. 20 shows the schematics of the harvesting system, employing the lowcost pump charge LT3588 (Linear Technology Inc. [31]), whereas the stepped output voltage is depicted in Fig. 21.

A significant limitation is due to the rectifying system. A common full bridge wave rectifier employs twice the diode voltage drop as shown in Fig 20. A large voltage drop cannot be tolerated, common MOS diodes or even discrete Schottky diodes must be avoided [32], preferring to these CMOS integrated active rectifier.

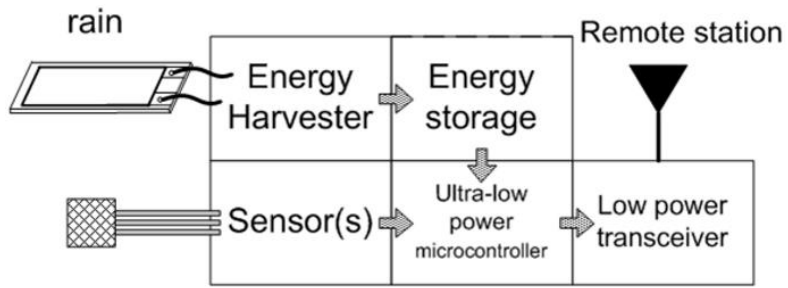

Environment: temperature, status, position,...

Fig. 19. Scheme of monitoring system fed by the raindrop energy harvester.

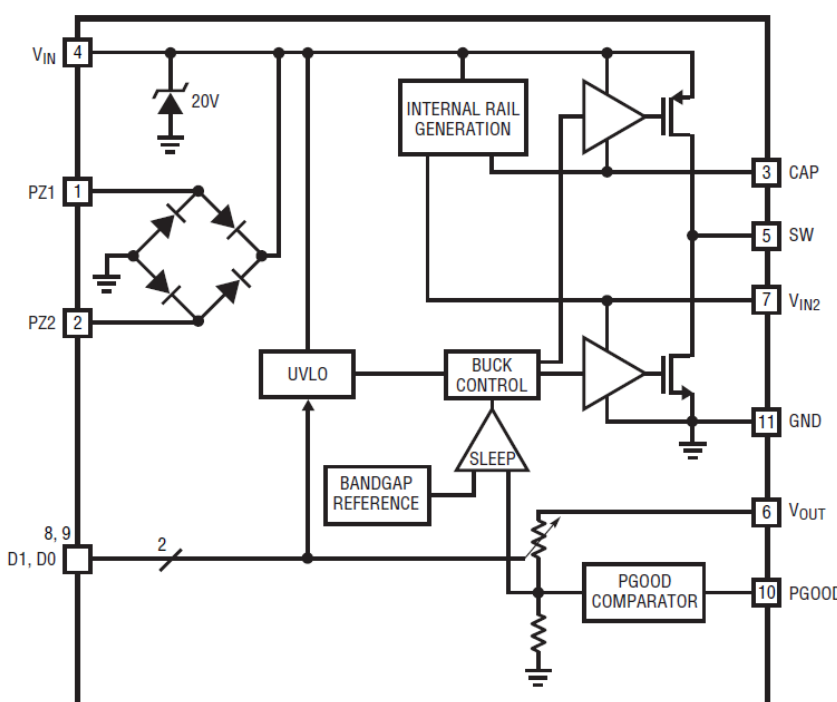

Fig. 20. Block diagram of the harvesting system employing the LTC3588. The transducer is connected between PZ1 and PZ2terminals.

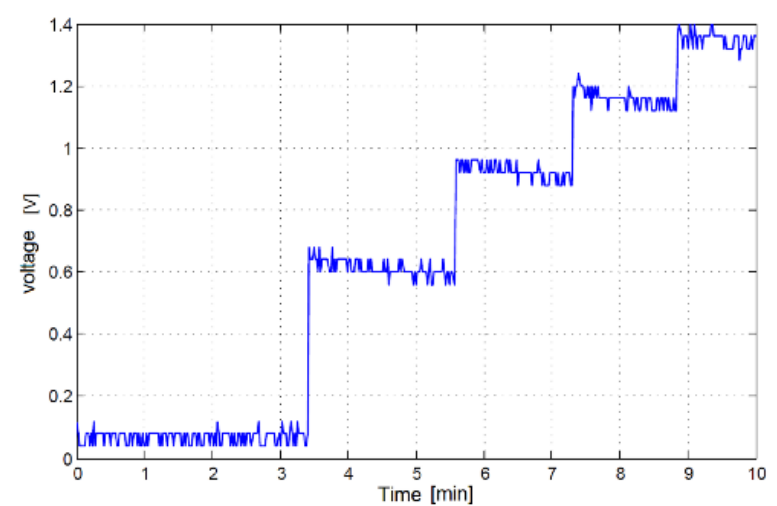

Fig. 21. Output voltage of the harvesting system.

In [32] a two stage rectifier is presented. First stage is called negative voltage converter and used to convert the negative half wave into a positive one. The second stage employs a common PMOS transistor connected as diode, or better, an active diode. Compared to a PMOS diode an active diode has a strongly reduced voltage drop, which is only some tens of millivolt.

Furthermore, in order to avoid full bridge diode rectifiers, in [33] a direct AC/DC converter is proposed. The system employs an interconnection of a boost and a buckboost converter. The boost converter steps up the voltage with same polarity as the source voltage $V_{\text {in }}$, while the buck-boost converter steps up the voltage with the opposite polarity of the source. The use of such system will allow to obtain an efficiency about $92 \%$, while for the full bridge rectifier system is about $50 \%$. Similarly [34] proposes the use of a back-boost in an automotive environment.

On the other hand, due to the engagement of more advanced conditioning circuits [35-39], or by modifying the shape of the cantilevers [40-41], ultra-low power applications are rapidly spreading.

The employment of such advanced systems will be the subject of future studies in which a comparison with the same strain will be performed.

\section{CONCLUSIONS}

The purpose of generating energy using rain as a source is to feed small and low consumption electronic components. However, the proposed experiments have confirmed the difficulty to guarantee a minimum power value needed for supply without the energy storage and this fact is clearly caused by the variability of the local precipitation intensity. The collision process itself presents very random behaviors, due to the occurrence of the "splashing" event, or even the inclination of the drop related to the impact surface, from the point where the impact takes place, from its speed as seen in the three axes and also from the presence of thin layers of water on the transducers.

The transduction efficiency is also affected by uncertainty, as the thin layer of water deposited on the transducer varies the mass and, consequentially, the inertia. The Arduino ${ }^{\circledR}$ platform, even if low-cost and open-source, 
has been a perfect aid for the above-mentioned experiment, obtaining 830 samples per second for all the five minutes detection period. The performances were improved for shorter detections reaching the peak of 895 samples per second for a study interval of 2 seconds. The same object can also be used to measure the kinetic energy of raindrops in order to predict soil erosion.

\section{ACKNOWLEDGMENTS}

This work was financially supported by MIUR Ministero dell'Istruzione dell'Università e della Ricerca (Italian Ministry of Education, University and Research) and by SDESLab (Sustainable Development and Energy Saving Laboratory) of the University of Palermo. Experimental measurements were carried out at the LEAP Laboratory Electrical Applications of the University of Palermo.

\section{REFERENCES}

[1] Benasciutti D. \& Moro L. (2010) Harvested power and sensitivity analysis of vibrating shoe-mounted piezoelectric cantilevers. Smart Material and Structures, vol. 19115011 doi:10.1088/0964-1726/19/11/115011

[2] Xiang H. J., Wang J. J., Shi Z. F. \& Zhang Z. W. (2013) . Theoretical analysis of piezoelectric energy harvesting from traffic induced deformation of pavements. Smart Materials and Structures Volume 22 (9), doi:10.1088/0964-1726/22/9/095024

[3] Van den Ende D. A., van de Wiel H. J., Groen W. A. \& van der Zwaag S. (2012). Direct strain energy harvesting in automobile tires using piezoelectric PZT-polymer compositesSmart Materials and Structures Volume 21

[4] Hobeck J. D. \& Inman D. J. (2012). Artificial piezoelectric grass for energy harvesting from turbulence-induced vibration, Smart Material and Structures, vol 21105024 doi:10.1088/0964$1726 / 21 / 10 / 105024$

[5] Jager T., Guigon R., Chaillout JJ \& Despesse G., (2008) . Harvesting raindrop energy: theory. Smart Materials and Structures Volume 17(1)

[6] Jager T., Guigon R., Chaillout JJ \& Despesse G., (2008) . Harvesting raindrop energy: experimental study. Smart Materials and Structures Volume 17 (1)

[7] Vatansever D., Hadimani R. L., Shah T. \& Siores E. (2011). An investigation of energy harvesting from renewable sources with PVDF and PZT, Smart Materials and Structures Volume 20 Number 5.

[8] Benasciutti D., Moro L. \& Gallina M.(2013). On the optimal bending deflection of piezoelectric scavengers. Journal of Intelligent Material Systems and Structures, vol. 24 (5), 627-639.

[9] Benasciutti D., Moro L., Zelenika S. \& Brusa E. (2010) . Vibration energy scavenging via piezoelectric bimorphs of optimized shapes. Microsyst Technol 16:657-668, DOI 10.1007/s00542-009-1000-5, Springer-Verlag

[10] Liang J. \& Liao WS (2011). Energy flow in piezoelectric energy harvesting systems. Smart Material and Structures, vol 20015005 doi:10.1088/0964-1726/20/1/015005

[11] Erturk A., \& Inman D. J. (2011). Piezoelectric energy harvesting. USA: John Wiley \& Sons.

[12] Roundy S. \& Wright P. K., (2004) . A piezoelectric vibration based generator for wireless electronics. Smart Materials and Structures 13 Volume 22 . (13) (2004) 1131-1142

[13] Viola F., Romano P., Miceli R. \& Acciari G. (2013). Harvesting rainfall energy by means of piezoelectric transducer.
International Conference on CLEAN ELECTRICAL POWER Renewable Energy Resources Impact 2013, June 11-13 2013, Alghero, Italy

[14] Viola F., Romano P., Miceli R. \& Spataro C. "Performances of rainfall energy harvester" 20th IMECO TC4, 2014 Symposium on Measurements of Electrical Quantities: Research on Electrical and Electronic Measurement for the Economic Upturn.

[15] Viola F., Romano P., Miceli R., Rainfall energy harvester, Innovative Materials and Systems for Energy Harvesting Applications, April 30, 2015, Pages 116-142

[16] Viola F., Romano P., Miceli R., Acciari G, Spataro C., Piezoelectric model of rainfall energy harvester, 2014 9th International Conference on Ecological Vehicles and Renewable Energies, EVER 2014

[17] Viola, F. , Romano, P., Miceli, R., Acciari, G., On the harvest of rainfall energy by means of piezoelectric transducer, Proceedings of 2013 International Conference on Renewable Energy Research and Applications, ICRERA 2013

[18] G. Acciari, M. Caruso, R. Miceli, L. Riggi, P. Romano, G. Schettino, F. Viola, Measuring Rain Energy with the Employment of "Arduino", Proceedings of 2016 International Conference on Renewable Energy Research and Applications, ICRERA 2016, Birmingham U.K., 20-23 november 2016

[19] Helseth, L.E. Wen, H.Z., Evaluation of the energy generation potential of rain cells, Department of Physics and Technology, University of Bergen, Allegaten 55, Bergen, Norway

[20] Lin ZH, Cheng G, Lee S, Pradel KC, Wang ZL. Harvesting water drop energy by sequential contact electrification and electrostatic induction process. Adv Mater 2014;26:4690e6.

[21]Chin-Hong Wong, Zuraini Dahari,Asrulnizam Abd Manaf, and Muhammad Azman Miskam,, "Harvesting Raindrop Energy with Piezoelectrics: a Review", Journal of ELECTRONIC MATERIALS, Vol. 44, No. 1, 2015

[22] Kok Gnee CHUA, Yew Fong HOR and Hee C. LIM, Raindrop Kinetic Energy Piezoelectric Harvesters and Relevant Interface Circuits: Review, Issues and Outlooks, Sensors \& Transducers, Vol. 200, Issue 5, May 2016, pp. 1-15

[[23] https://www.arduino.cc/

[24] Caruso, M., Di Tommaso, A.O., Miceli, R., Galluzzo, G.R., Romano, P., Schettino, G., Viola, F., Design and experimental characterization of a low-cost, real-time, wireless AC monitoring system based on ATmega 328P-PU microcontroller, AEIT International Annual Conference: A Sustainable Development in the Mediterranean Area, AEIT 2015; Basilica San Giovanni Maggiore Naples; Italy; 14 October 2015 through 16 October 2015;

[25] M. Caruso, R. Miceli, P. Romano, G. Schettino, C. Spataro and F. Viola, A Low-cost, Real-time Monitoring System for PV Plants based on ATmega 328P-PU Microcontroller, proceedings of 37th International Telecommunications Energy Conference 2015, Intelec, 18-22 October, Osaka, Japan

[26] Perera K. C. R. , Sampath B.G., Dassanayake V.P.C. \& Hapuwatte B. M., Harvesting of Kinetic Energy of the Raindrops, International Journal of Mathematical, Computational, Physical and Quantum Engineering Vol.8 (2), 2014

[27] Wong Chin-Hong, Dahari, Z. ; Abd Manaf, A. ; Sidek, O., Miskam, M.A. \& Mohamed, J.J., Simulation of piezoelectric raindrop energy harvester, TENCON Spring Conference, 2013 IEEE, 465 - 469, 2013.

[28] Datasheet of Mide Volture V22B, retrieved from http://www.mide.com/index.php

[29] Datasheet of MEAS LDT1-028K., retrieved from http://www.meas-spec.com 
[30] Pinna L., Dahiya R., \&Valle M., (2009), SPICE Model for Piezoelectric Bender Generators, 16th IEEE International Conference on Electronics, Circuits, and Systems, ICECS Hammamet,Tunisia, 13-16 dec 2009.

[31] Datasheet of linear technology LT3588. http://www.linear.com

[32] C. Peters, J. Handwerker, D. Maurath and Y. Manoli, "A Sub$500 \mathrm{mV}$ Highly Efficient Active Rectifier for Energy Harvesting Applications," in IEEE Transactions on Circuits and Systems I: Regular Papers, vol. 58, no. 7, pp. 1542-1550, July 2011, doi: 10.1109/TCSI.2011.2157739

[33] C. Y. Hsieh, M. Moallem and F. Golnaraghi, "Bridgeless converter with input resistance control for low-power energy harvesting applications," in IET Power Electronics, vol. 8, no. 5, pp. 822-830, 5 2015.doi: 10.1049/iet-pel.2014.0283

[34] C. Y. Hsieh, M. Moallem and F. Golnaraghi, "A simple Bidirectional bridgeless AC/DC buck-boost converter for automotive energy harvesting," IECON 2014 - 40th Annual Conference of the IEEE Industrial Electronics Society, Dallas, TX, 2014, pp. 19371943. doi: 10.1109/IECON.2014.7048767

[35] A. Álvarez-Carulla, J. Colomer-Farrarons, J. López-Sánchez and P. Miribel-Català, "Piezoelectric harvester-based self-powered adaptive circuit with wireless data transmission capability for structural health monitoring," 2015 Conference on Design of Circuits and Integrated Systems (DCIS), Estoril, 2015, pp. 1-6.doi: 10.1109/DCIS.2015.7388594

[36] J. Y. Lei and G. A. Rincón-Mora, "Maximum power-point extraction of small switched-inductor piezoelectric harvesters," 2014 21st IEEE International Conference on Electronics, Circuits and Systems (ICECS), Marseille, 2014, pp. 112-115. doi: 10.1109/ICECS.2014.7049934

[37] R. D'hulst, T. Sterken, R. Puers, G. Deconinck and J. Driesen, "Power Processing Circuits for Piezoelectric Vibration-Based Energy Harvesters," in IEEE Transactions on Industrial Electronics, vol. 57, no. 12, pp. 4170-4177, Dec. 2010. doi: 10.1109/TIE.2010.2044126

G. A. Rincón-Mora and S. Yang, "Tiny Piezoelectric Harvesters: Principles, Constraints, and Power Conversion," in IEEE Transactions on Circuits and Systems I: Regular Papers, vol. 63, no. 5, pp. 639-649, May 2016. doi: 10.1109/TCSI.2016.2555249

[38]A. V. Korshunov and P. S. Volobuev, "Exploring of equivalent circuit model of piezoelectric energy harvesters for ultra lowpower devices," 2015 IEEE East-West Design \& Test Symposium (EWDTS), Batumi, 2015, pp. 1-4. doi: 10.1109/EWDTS.2015.7493115

[39] G. Acciani, F. Di Modugno, E. Mininno and P. Montegiglio, "Multi-physics simulation of a wind piezoelectric energy harvester validated by experimental results," 201617 th International Conference on Thermal, Mechanical and Multi-Physics Simulation and Experiments in Microelectronics and Microsystems (EuroSimE), Montpellier, 2016, pp. 1-6.

[40] D. S. Kwon, H. J. Ko and J. Kim, "Piezoelectric and electromagnetic hybrid energy harvester using two cantilevers for frequency up-conversion," 2017 IEEE 30th International Conference on Micro Electro Mechanical Systems (MEMS), Las Vegas, NV, 2017, pp. 49-52 doi: 10.1109/MEMSYS.2017.7863336

[41] A. Iqbal and F. Mohd-Yasin, "Comparison of seven cantilever designs for piezoelectric energy harvester based On Mo/AlN/3C$\mathrm{SiC}, 2015$ IEEE Regional Symposium on Micro and Nanoelectronics (RSM), Kuala Terengganu, 2015, pp. 1-4. doi: 10.1109/RSM.2015.7354987 